\title{
Editorial: Gut Microbial Response to Host Metabolic Phenotypes
}

\author{
Jie Yin ${ }^{1}$, Yong Su ${ }^{2 *}$ and Hui Han ${ }^{3 *}$ \\ ${ }^{1}$ College of Animal Science and Technology, Hunan Agricultural University, Changsha, China, ${ }^{2}$ College of Animal Science and \\ Technology, Nanjing Agricultural University, Nanjing, China, ${ }^{3}$ State Key Laboratory of Animal Nutrition, Institute of Animal \\ Science, Chinese Academy of Agricultural Sciences, Beijing, China
}

Keywords: gut microbiota, metabolism, disease, probiotics, diets

\section{Editorial on the Research Topic}

\section{Gut Microbial Response to Host Metabolic Phenotypes}

It is increasingly apparent that gut microbiota perform functions crucial to the host, such as regulating host physiology and influencing host health (1-3). Using fecal bacteria transplantation technology, Wu et al. found that fecal microbiota from obese Jinhua pigs and lean Landrace pigs exert different lipid metabolic phenotypes. Zheng et al. also witnessed gut microbial alterations in high fat diet-fed mice, with a high ratio of Firmicutes to Bacteroidetes and abundance of Allobaculum. Although this Research Topic failed to receive any papers about the role of gut microbiota in amino acid metabolism, nucleic acid metabolism, and carbohydrate metabolism, other reports have confirmed these functions $(4,5)$.

Currently, the gut microbiota is attracting much interest due to its role in maintaining host health and its association with all aspects of health and diseases. In this Research Topic, gut microbial disorders are screened in persistent atrial fibrillation patients ( $\mathrm{Xu}$ et al.) renal cell

OPEN ACCESS

Edited and reviewed by: Christophe Lacroix,

ETH Zürich, Switzerland

*Correspondence:

Yong Su

yong.su@njau.edu.cn

Hui Han

hanhui16@mails.ucas.ac.cn

Specialty section:

This article was submitted to Nutrition and Microbes,

a section of the journal

Frontiers in Nutrition

Received: 18 November 2021 Accepted: 13 December 2021 Published: 13 January 2022

Citation:

Yin J, Su Y and Han H (2022) Editorial: Gut Microbial Response to Host Metabolic Phenotypes.

Front. Nutr. 8:817501.

doi: 10.3389/fnut.2021.817501 carcinoma metastasis patients (Dai et al.), and a spinal cord injury animal model (Rong et al.). $\mathrm{Xu}$ et al. thoroughly discussed the taxonomic and functional characteristics of the gastrointestinal microbiota and demonstrated the profound relationship between gastrointestinal microbiota and metabolic disorders in ruminants. Together, these results further confirmed the role of gut microbiota in disease occurrence and development and manipulation of gut microbiota might, therefore, be considered a potential target for treating diseases.

Indeed, various disease treatment measures include gut microbial improvement, such as dietary probiotics (6). For example, Zhang et al. reported that dietary Lactobacillus acidophilus ATCC 4356 improved gut microbiota distribution and alleviated renal ischemia-reperfusion injury. Similarly, beneficial effects of Lactobacillus have been identified in lumbar disc herniation (Wang et al.), hypercholesterolemic golden hamsters (Yang et al.), asthma, and Clostridium perfringens infection (Wang et al.). In animal production, the gut is generally disturbed by weaning stress, dietary toxins, and pathogen infections, thus dietary probiotics have been widely introduced to maintain a healthy gut and guarantee higher production performance (7). Lactococcus lactis, in this Research Topic, has been identified to improve gut function in piglets (Yu et al.). However, probiotics are not limited to Lactobacillus, some species of Bifidobacterium, Escherichia coli, Enterococcus, and Saccharomyces have long been used as probiotics to alleviate various diseases by changing gut microbiota compositions.

Gut microbiota diversity and compositions are highly associated with dietary fluctuations. Thus, dietary manipulation has also been used to target gut microbiota to regulate host physiology and metabolism. In this Research Topic, Qian et al. found that dietary dried citrus peel (Chenpi) improved gut microbiota compositions in high fat diet-fed mice. Li et al. concluded that maternal fiber nutrition during pregnancy regulated the health of offspring, and the response of the maternal 
intestinal microbes played an important role in intervening in the phenotype of sows and neonatal piglets. Dietary protein and amino acids are the main factors shaping gut microbiota (8), $\mathrm{Fu}$ et al. also reported a role of tryptophan in gut microbiota. Besides, vitamin K2 (Liu et al.), $\beta$-carotene (Yuan et al.), olive fruit extracts (Wang et al.), bovine lactoferrin (Wang et al.), and $\beta$-sitosterol (Yu et al.) have been reported to shape gut microbiota compositions in this Research Topic.

How does gut microbiota affect host physiology and metabolism? Hou et al. showed that the gut-liver FXR-FGF19 axis is involved in Lactobacillus delbrueckii-promoted ileal bile acid deconjugation. In our previous studies, we found that gut microbiota-derived metabolites are highly associated with host metabolic reprograming (9-12). Furthermore, bacterial microRNA, bacteriocin, and microbiota sensing pathways have also been identified to be involved in the relationship between gut microbiota and host metabolism $(13,14)$. However, with the focus on the detailed mechanism by which gut microbiota influence host metabolism, much still needs to be elucidated.

In summary, papers from the current Research Topic screened the gut microbiota dysbiosis in various diseases and reported the beneficial roles of dietary probiotics and other active components in the improvement of gut microbiota. Despite the progress made in understanding the relationship between gut microbiota and host metabolism, there are a number of prominent research avenues that remain to be explored. For example, what are the

\section{REFERENCES}

1. Yin J, Li YY, Han H, Ma J, Liu G, Wu X, et al. Administration of exogenous melatonin improves the diurnal rhythms of the gut microbiota in mice fed a high-fat diet. mSystems. (2020) 5:e00002-20. doi: 10.1128/mSystems.00002-20

2. Tian M, Chen JM, Liu JX, Chen F, Guan WT, Zhang HS. Dietary fiber and microbiota interaction regulates sow metabolism and reproductive performance. Anim Nutr. (2020) 6:397-403. doi: 10.1016/j.aninu.2020.10.001

3. Ma J, Zheng YM, Tang WJ, Yan WX, Nie HF, Fang J, et al. Dietary polyphenols in lipid metabolism: a role of gut microbiome. Anim Nutr. (2020) 6:4049. doi: 10.1016/j.aninu.2020.08.002

4. Agus A, Planchais J, Sokol H. Gut microbiota regulation of tryptophan metabolism in health and disease. Cell Host Microbe. (2018) 23:71624. doi: 10.1016/j.chom.2018.05.003

5. Fan Y, Pedersen O. Gut microbiota in human metabolic health and disease. Nat Rev Microbiol. (2021) 19:55-71. doi: 10.1038/s41579-020-0433-9

6. Azad MAK, Gao J, Ma J, Li TJ, Tan B, Huang XG, et al. Opportunities of prebiotics for the intestinal health of monogastric animals. Anim Nutr. (2020) 6:379-88. doi: 10.1016/j.aninu.2020.08.001

7. Azad MAK, Sarker M, Li T, Yin J. Probiotic species in the modulation of gut microbiota: an overview. Biomed Res Int. (2018) 2018:9478630. doi: 10.1155/2018/9478630

8. Yin J, Ma J, Li YY, Ma XK, Chen JS, Zhang HH, et al. Branched-chain amino acids, especially of leucine and valine, mediate the protein restricted response in a piglet model. Food Funct. (2020) 11:1304-11. doi: 10.1039/C9FO01757G

9. Yin J, Li YY, Han H, Chen S, Gao J, Liu G, et al. Melatonin reprogramming of gut microbiota improves lipid dysmetabolism in high-fat diet-fed mice. $J$ Pineal Res. (2018) 65:e12524. doi: 10.1111/jpi.12524

10. Duan YH, Zhong YZ, Xiao H, Zheng CB, Song B, Wang WL, et al. Gut microbiota mediates the protective effects of dietary beta-hydroxy-betamethylbutyrate (HMB) against obesity induced by high-fat diets. Faseb J. (2019) 33:10019-33. doi: 10.1096/fj.201900665RR molecular and physiological links between the gut microbiota and host metabolism at the epigenetic, transcriptome, and proteome levels? Gut microbiota is changed in various pathologic conditions and microbial biomarkers need to be screened in specific metabolic diseases. Additionally, dietary manipulation is widely used to maintain a healthy gut microbiota composition, and the interaction between diets, gut microbiota, and host metabolism will be an important area of future research.

\section{AUTHOR CONTRIBUTIONS}

All authors listed have made a substantial, direct, and intellectual contribution to the work and approved it for publication.

\section{FUNDING}

This study was supported by the National Natural Science Foundation of China (32172761) and the Young Elite Scientists Sponsorship Program by CAST (2019-2021QNRC001).

\section{ACKNOWLEDGMENTS}

We would like to thank all authors for their papers and the reviewers for the painstaking care taken in helping improve the clarity of the manuscript.
11. Han H, Yi B, Zhong R, Wang M, Zhang S, Ma J, et al. From gut microbiota to host appetite: gut microbiota-derived metabolites as key regulators. Microbiome. (2021) 9:162. doi: 10.1186/s40168-021-01093-y

12. Han $H$, Jiang $Y$, Wang M, Melaku M, Liu L, Zhao Y, et al. Intestinal dysbiosis in nonalcoholic fatty liver disease (NAFLD): focusing on the gut-liver axis. Crit Rev Food Sci Nutr. (2021) 18:1-18. doi: 10.1080/10408398.2021.1966738

13. Nicholson JK, Holmes E, Kinross J, Burcelin R, Gibson G, Jia W, et al. Host-gut microbiota metabolic interactions. Science. (2012) 336:1262-7. doi: $10.1126 /$ science. 122 3813

14. Visconti A, Le Roy CI, Rosa F, Rossi N, Martin TC, Mohney $\mathrm{RP}$, et al. Interplay between the human gut microbiome and host metabolism. Nat Commun. (2019) 10:4505. doi: 10.1038/s41467-019-12 $476-\mathrm{z}$

Conflict of Interest: The authors declare that the research was conducted in the absence of any commercial or financial relationships that could be construed as a potential conflict of interest.

Publisher's Note: All claims expressed in this article are solely those of the authors and do not necessarily represent those of their affiliated organizations, or those of the publisher, the editors and the reviewers. Any product that may be evaluated in this article, or claim that may be made by its manufacturer, is not guaranteed or endorsed by the publisher.

Copyright (c) 2022 Yin, Su and Han. This is an open-access article distributed under the terms of the Creative Commons Attribution License (CC BY). The use, distribution or reproduction in other forums is permitted, provided the original author(s) and the copyright owner(s) are credited and that the original publication in this journal is cited, in accordance with accepted academic practice. No use, distribution or reproduction is permitted which does not comply with these terms. 